\title{
An optical and near IR study of the old open cluster NGC $2141^{\star}$
}

\author{
G. Carraro ${ }^{1}$, S. M. Hassan ${ }^{2}$, S. Ortolani ${ }^{1}$, and A. Vallenari ${ }^{3}$ \\ 1 Dipartimento di Astronomia, Universitá di Padova, vicolo dell'Osservatorio 5, 35122 Padova, Italy \\ 2 National Research Institute of Astronomy and Geophysics, Helwan, Cairo, A.R.E. \\ 3 Osservatorio Astronomico di Padova, vicolo Osservatorio 5, 35122, Padova, Italy \\ e-mail: carraro, ortolani, vallenari@pd.astro.it
}

Received 8 February 2001 / Accepted 9 April 2001

\begin{abstract}
We report CCD optical ( $B$ and $V$ passbands) and near IR ( $J$ and $K$ bands) observations in the region of the old open cluster NGC 2141. By combining the two sets of photometry (500 stars in common) we derive new estimates of the cluster's fundamental parameters. We confirm that the cluster is 2.5 Gyrs old, but, with respect to previous investigations, we obtain a slightly larger reddening $(E(B-V)=0.40)$, and a slightly shorter distance $(3.8 \mathrm{kpc})$ from the Sun. Finally, we present the Luminosity Function (LF) in the $V$ band, which is another age indicator. We provide a good fit for the age range inferred from isochrones by assuming the Kroupa et al. (1993) IMF up to $M_{V}=5.0$. We interpret the disagreement at fainter magnitudes as evidence of mass segregation.
\end{abstract}

Key words. open clusters and associations: NGC 2141: individual - techniques: photometric

\section{Introduction}

In this paper we continue a series dedicated to the presentation of near-infrared photometry (in $J$ and $K$ pass-bands) for northern galactic open clusters. We already reported observations of the very young open clusters NGC 1893 and Berkeley 86 (Vallenari et al. 1999), the old clusters Berkeley 17 and Berkeley 18 (Carraro et al. 1999a), the intermediate age clusters IC 166 and NGC 7789 (Vallenari et al. 2000) and King 5 (Carraro \& Vallenari 2000). Here we combine optical $B$ and $V$ and near-infrared $J$ and $K$ photometry for NGC 2141, a faint old open cluster which has not received much attention in the past.

NGC 2141 is located close to the galactic plane in the anticenter direction at $l=198.75$ and $b=-5 \circ 79$, and it is also designated as OCL 487 (Lund 203) and C0600+104 by IAU. Its diameter is estimated to be about $10^{\prime}$.

A preliminary investigation was conducted by Burkhead et al. (1972) who obtained photographic and photoelectric $U B V$ photometry for about 300 stars with the aim of assessing whether the cluster is very old. This study revealed that NGC 2141 is a cluster of late inter-

Send offprint requests to: G. Carraro,

e-mail: carraro@pd.astro.it

* Based on observations taken at ESO La Silla and TIRGO. Table 2 is only available in electronic form at the CDS via anonymous ftp to cdsarc.u-strasbg.fr (130.79.128.5) or via

http://cdsweb.u-strasbg.fr/cgi-bin/qcat?J/A+A/372/879

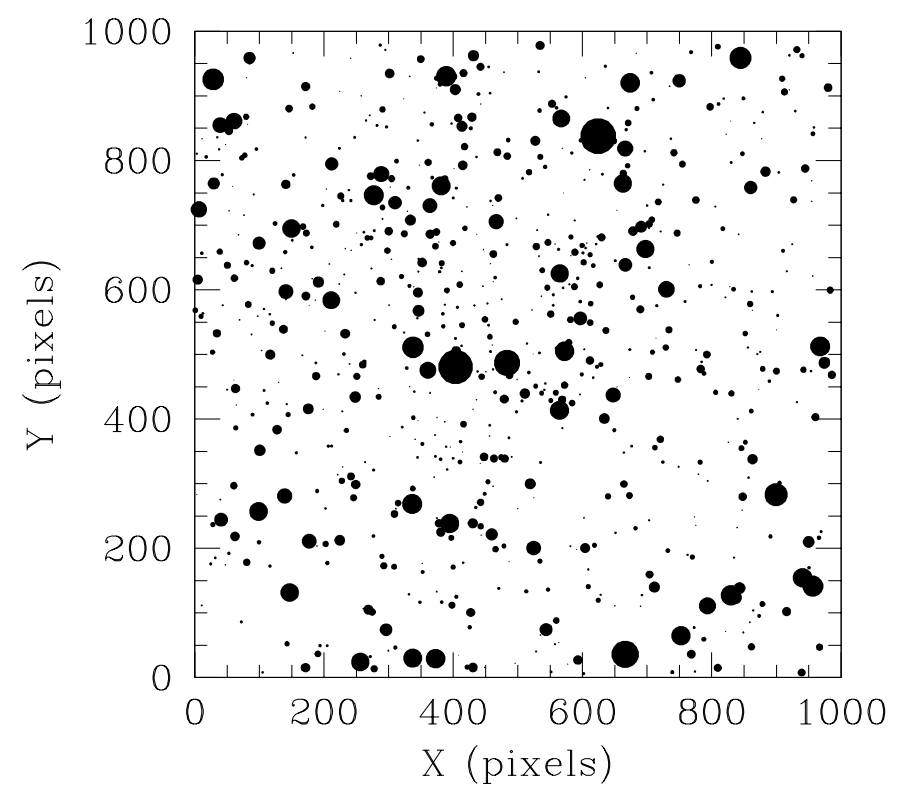

Fig. 1. The field covered in the region of NGC 2141 by the optical photometry. The mosaic of the four fields observed in the IR covers almost the same region. North is up, East on the left.

mediate age, $4.4 \mathrm{kpc}$ distant from the sun, and with a reddening $E(B-V)=0.30$.

The metal abundance of NGC 2141 has been determined several times in the past. Janes (1979) obtained a value $[\mathrm{Fe} / \mathrm{H}]=-0.54 \pm 0.42$ from DDO photometry, while Geisler (1987) obtained $[\mathrm{Fe} / \mathrm{H}]=-0.63 \pm 0.15$ from 
Table 1. Observation Log for NGC 2141.

\begin{tabular}{|c|c|c|c|c|c|c|}
\hline \multirow[t]{2}{*}{ Run } & \multirow{2}{*}{$\begin{array}{c}\alpha \\
(2000)\end{array}$} & \multirow{2}{*}{$\begin{array}{c}\delta \\
(2000)\end{array}$} & \multirow[t]{2}{*}{ Date } & \multicolumn{2}{|c|}{ Exposure Times (s) } & \multirow[t]{2}{*}{ Field of view } \\
\hline & & & & $J$ & $K$ & \\
\hline Tirgo & 060258.2 & 102639 & Oct., 23,1997 & 480 & 680 & $7.5 \times 7.5$ \\
\hline & & & & $\mathrm{B}$ & $\mathrm{V}$ & \\
\hline $2.2 \mathrm{~m} \mathrm{ESO} / \mathrm{MPI}$ & $06 \quad 02 \quad 58.2$ & 102639 & Dec., 8,1991 & 1200 & 4200 & $5^{\prime} .7 \times 5^{\prime} .7$ \\
\hline
\end{tabular}

Washington photometry. Finally, by using medium resolution spectroscopy of six giant stars, Friel \& Janes (1993) found $[\mathrm{Fe} / \mathrm{H}]=-0.39 \pm 0.11$.

The kinematics of NGC 2141 has been studied by Friel et al. (1989) and Friel (1989). This latter study (5 stars) indicates that the radial velocity is $V_{\mathrm{r}}=43 \pm 6 \mathrm{~km} \mathrm{~s}^{-1}$. Individual radial velocities for 15 stars have been obtained by Minniti (1995). This survey has only one star in common with Friel (1989) and the radial velocity estimate is in agreement, suggesting that most of Minniti (1995) stars might probably be cluster non members. A much deeper investigation is required to isolate cluster members in NGC 2141.

More recently, Rosvick (1995) published optical VI photometry of 3561 stars in NGC 2141 together with $J H$ infrared photometry for a handful of stars. This is the first comprehensive study of NGC 2141. The author infers, by comparison with half solar $(Z=0.006)$ isochrones, an age of $2.5 \mathrm{Gyr}$, a distance of $4.2 \mathrm{kpc}$ and a reddening $E(B-V)=0.35$.

In this paper, we combine IR $J K$ (765 stars) and optical $B V$ (1073 stars) photometry to obtain new estimates of the cluster's fundamental parameters. The layout of the paper is as follows: Sect. 2 is devoted to the presentation of data acquisition and reduction; Sect. 3 deals with the morphology of the Color Magnitude Diagrams (CMDs) for different pass-bands; Sect. 4 concerns the derivation of the cluster metallicity, whereas Sect. 5 deals with the estimate of color excess. Section 6 is devoted to inferring the age and distance, while in Sect. 7 we discuss the Luminosity Function. Finally our conclusions are summarized in Sect. 8.

\section{Observations and data reduction}

\subsection{TIRGO observations}

$J(1.2 \mu \mathrm{m})$ and $K(2.2 \mu \mathrm{m})$ photometry of NGC 2141 was obtained with the $1.5 \mathrm{~m}$ Gornergrat Infrared Telescope (TIRGO) equipped with the Arcetri Near Infrared Camera (ARNICA) in October 1997. ARNICA is based on a NICMOS3 $256 \times 256$ pixels array (gain $=20 \mathrm{e}^{-} / \mathrm{ADU}$, read-out noise $=50 \mathrm{e}^{-}$, angular scale $=1^{\prime \prime} /$ pixel, and $4^{\prime} \times 4^{\prime}$ field of view). More details about the observational equipment and infrared camera, and the reduction procedure can be found in Carraro et al. (1999a). Through each filter, 4 partially overlapping images of each field were obtained, covering a total field of view of about $7.5 \times 7^{\prime} .5$, in short exposures to avoid sky saturation.
The log of the observations is presented in Table 1 , where the center of the observed field and the total exposure times are given. The night was photometric with a seeing of $1^{\prime \prime}-1.5^{\prime \prime}$. Figure 1 presents the final mosaic of the 4 frames for NGC 2141 in the $K$ passband.

The conversion of the instrumental magnitude $j$ and $k$ to the standard $J, K$ was made using stellar fields of standard stars taken from the Hunt et al. (1998) list. About 10 standard stars per night were used.

The relations in usage per $1 \mathrm{~s}$ exposure time are:

$J=j+19.51+k_{J} \times 1.24$

$K=k+18.94+k_{K} \times 1.24$

where $k_{J}$ and $k_{K}$ (the extinction coefficients, in magnitude per airmass) are 0.25 and 0.10 , respectively. The standard deviation of the zero points are of $0.03 \mathrm{mag}$ for the $J$ and 0.04 for the $K$ magnitude. This error is due only to the linear interpolation of the standard stars. The calibration uncertainty is dominated by the error due to the correction from aperture photometry to PSF fitting magnitude. The standard stars used for the calibration do not cover the entire color range of the data, because of the lack of stars redder than $(J-K) \sim 0.8$. From our data, no color term is found for $K$ mag, whereas we cannot exclude it for the $J$ magnitude. Taking all into account, we estimate that the total error on the calibration is about $0.1 \mathrm{mag}$ in both $J$ and $K$ pass-bands (see Vallenari et al. 2000 for additional details).

\section{2. $2.2 \mathrm{~m} \mathrm{ESO/MPI} \mathrm{observations}$}

NGC 2141 was observed with the $2.2 \mathrm{~m}$ ESO/MPI telescope at La Silla. The focal reducer ESO EFOSC 2 camera was used, equipped with the Thompson UV coated $1000 \times 1000$ pixels CCD ESO \# 19. The chip has 19 micron pixel size, corresponding to $0^{\prime \prime} 34 /$ pixel projected on the sky. The total field of view is about $5 ! 7 \times 5 ! 7$. The observations were carried out in 1991 December 8 (see Table 1 for details). The night was photometric with an average seeing of 1.5 . Several standard field stars from Landolt (1992) were observed during the same night, from which the following color equations have been derived:

$$
\begin{aligned}
& V=v+25.75+0.05 \times(B-V) \\
& B=b+26.11+0.21 \times(B-V) .
\end{aligned}
$$

After the standard pre-processing, instrumental magnitudes have been extracted using DAOPHOT II and the 


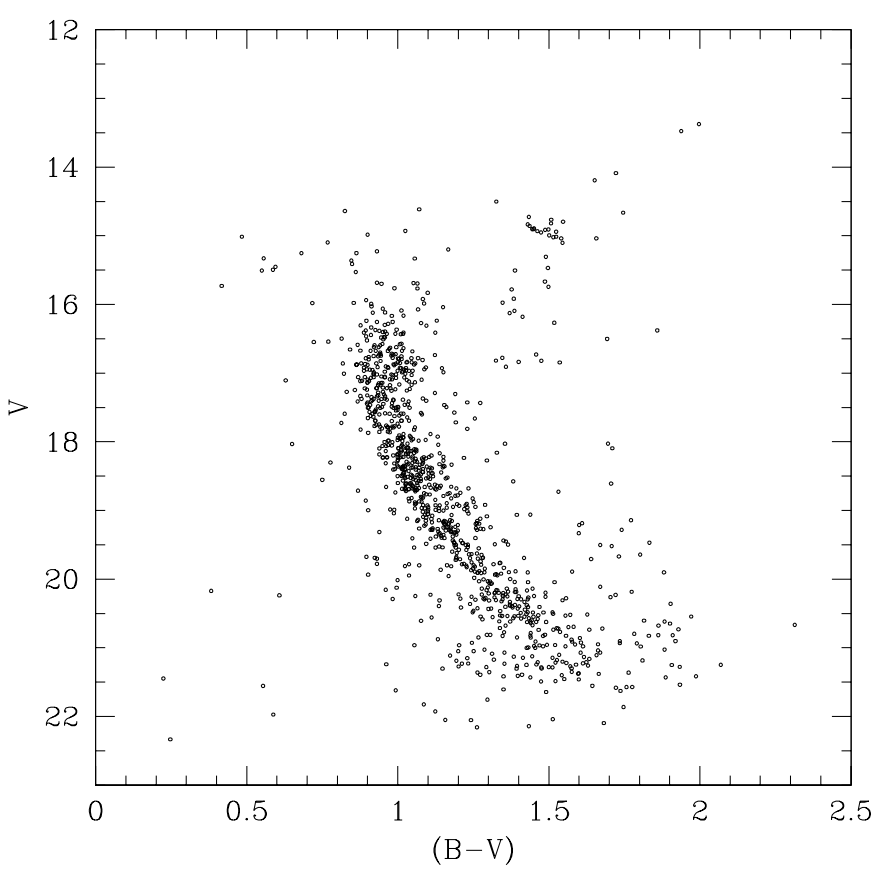

Fig. 2. The CMD of NGC 2141 derived from optical photometry.

accompanying ALLSTAR program in the MIDAS environment. Since the field is relatively crowding free, the aperture corrections have been directly computed on the original frames, giving the coefficient within a $0.02 \mathrm{mag}$ spread. The final errors in the zero points amount to 0.02 both in $B$ and in $V$. The comparison of 5 stars in common with Burkhead et al. (1972) yields:

$V_{\mathrm{BBH}}-V_{\mathrm{CHOV}}=0.01 \pm 0.01$

$(B-V)_{\mathrm{BBH}}-(B-V)_{\mathrm{CHOV}}=0.02 \pm 0.02$

where BBH and $\mathrm{CHOV}$ refer to Burkhead et al. (1972) and the present work, respectively.

We estimated the photometric errors by means of experiments with artificial stars (Carraro \& Ortolani 1994), obtaining errors of $0.02,0.04$ and 0.08 at $V=18,19$ and $20 \mathrm{mag}$, respectively. Another estimate of the photometric errors derives from the MS natural width. At the same above magnitude levels we found dispersions in color of $0.11,0.16$ and 0.20 . These latter values clearly also take into account the effect of unresolved binaries (see the sequence rightwards of the MS in Figs. 2 and 3) and possible variable reddening.

All the photometric data are given in Table 2.

\section{The Color Magnitude Diagram}

In this section we discuss separately the CMDs obtained from the optical and near IR photometry.

\subsection{The optical CMD}

The CMD derived from optical photometry is shown in Fig. 2.

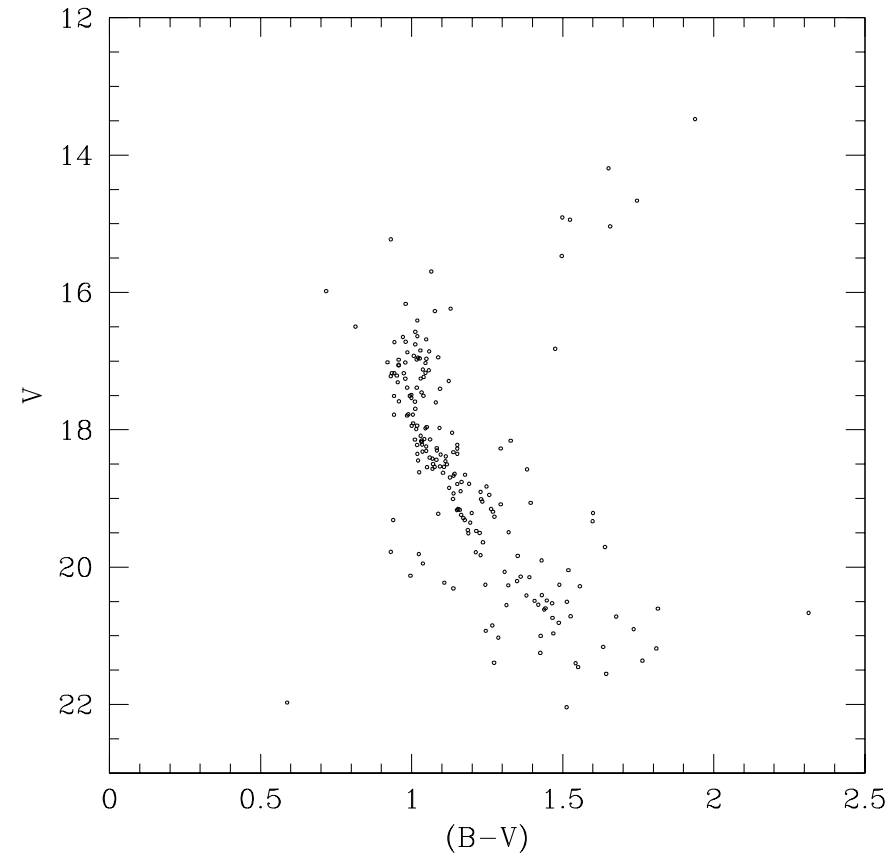

Fig. 3. The CMD of NGC 2141 derived from optical photometry by considering only stars within 1.2 arcmin from the cluster center. Note the presence of a parallel sequence red-wards to the MS, which we ascribe to the presence of unresolved binaries.

The global morphology resembles the CMD of an intermediate age or old open cluster like NGC 7789 (Girardi et al. 2000a), IC 4651 (Bertelli et al. 1992) and NGC 2204 (Kassis et al. 1997), say a cluster whose age is between NGC 752 (1.7 Gyr) and M 67 (4.0 Gyr) (Carraro et al. 1999b). The MS extends down to $V \approx 22$, and the Turn Off point (TO) is situated at $V=15-16.5$, $(B-V)=0.9$, with a few star spreading towards brighter regions $(V \approx 16)$. The concentration of stars in the red region of the diagram (at $V \approx 15.0,(B-V) \approx 1.5$ ) represents the Red Giant (RG) clump of core He-burning stars. Note the tilt and the extension of the clump in color, which can be ascribed to a possible spread in metallicity or in age, or to the presence of some differential reddening. The most reasonable explanation is a metallicity effect. Indeed, the MS is rather thin up to the limiting magnitude, thus ruling out a significant age dispersion. A secondary sequence of unresolved binary stars is visible on the red side of the MS. This is more evident from Fig. 3, where only the core of the cluster is considered. By counting the number of stars belonging to the two different sequences, we find that the minimum binary percentage is $30 \%$. Finally, the Herzsprung gap is clearly defined, while the sub-giant and RG branch are scarcely populated.

The population of stars on the right side of the MS and above the TO probably belongs to the field or they might be blue stragglers which are members of the cluster. The field star contamination is not very high. The MS is much thinner than in Rosvick (1995) even after the field star decontamination (see her Fig. 5). This rules out the 


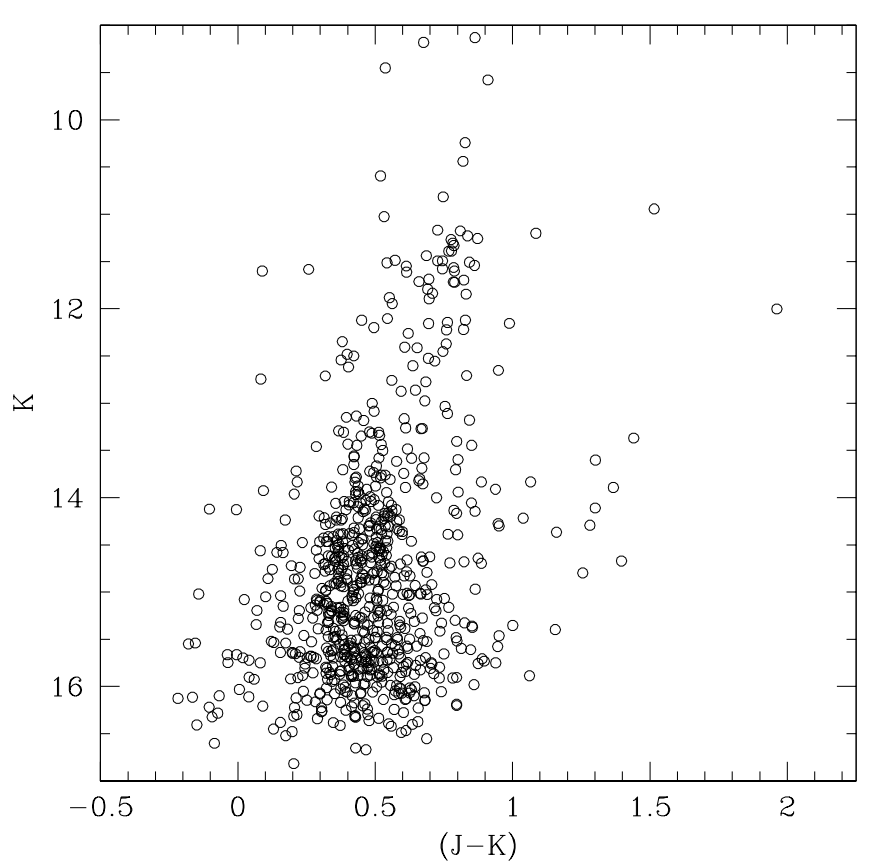

Fig. 4. The CMD of NGC 2141 derived from near IR photometry.

presence of a significant differential reddening across the $5^{\prime} \times 5^{\prime}$ region covered by the present photometry.

\subsection{The near IR CMD}

The CMD from IR photometry is shown in Fig. 4. The MS extends down to $K=16.5$, and the TO is located at $K \approx 14.5,(J-K) \approx 0.40$. The region above the $\mathrm{TO}$ might contain some interlopers (see Fig. 2).

The stars at $K \approx 11.5,(J-K) \approx 0.8$ might represent the clump of core He burners. The cluster is indeed faint, and the MS gets rather wide down to $K \approx 15$. The MS is much larger than in the optical CMD, due to the larger photometric errors we have in the IR photometry (see Sect. 2). Apparently there is no Herzsprung gap, and the RGB is scarsely populated, but sufficiently evident to be used to estimate cluster metallicity (see next section).

\section{Metallicity}

The CMD diagram in the IR allows us to derive an independent estimate of the cluster abundance by using a photometric method (Tiede et al. 1997). This method correlates the slope of the RGB, defined as $\Delta(J-K) / \Delta K$ with the cluster metallicity, measured by the index $[\mathrm{Fe} / \mathrm{H}]$, and for open clusters, the relation reads:

$$
[\mathrm{Fe} / \mathrm{H}]=-1.639-14.243 \times(\mathrm{GB} \text { slope }) .
$$

This method has already been applied by us in the study of NGC 7789 (Vallenari et al. 2000).

To find the RGB slope we performed a least squares fit to the RGB stars. This yields a RGB slope $\Delta(J-K) / \Delta K$

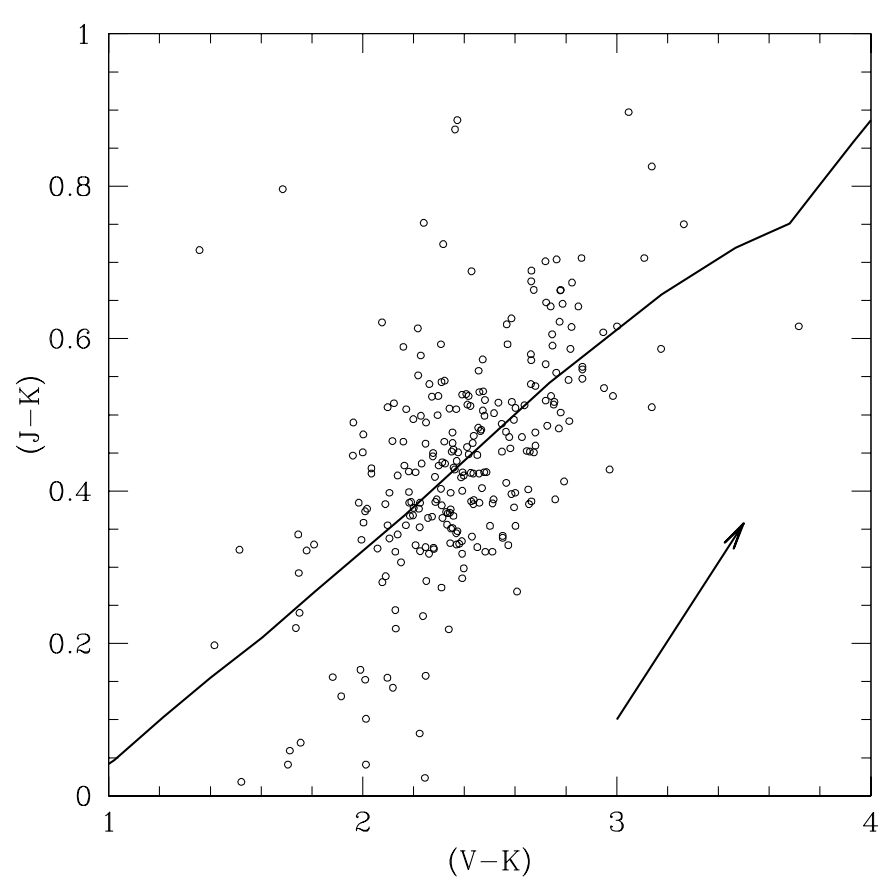

Fig. 5. Two color diagram for MS stars in NGC 2141. The solid line is a Zero Age MS for $[\mathrm{Fe} / \mathrm{H}]=-0.43$. In the lower right corner the reddening vector is shown. See text for details.

of $-0.085 \pm 0.006$. By using the relation (3), we obtain $[\mathrm{Fe} / \mathrm{H}]=-0.43 \pm 0.07$. The reported error is derived as:

$\Delta([\mathrm{Fe} / \mathrm{H}])=14.243 \times \Delta(\mathrm{GB}$ slope $)$

and has to be considered as an optimistic estimate, since it does not take into account the uncertainties in the coefficients of Eq. (3), and the sensitivity of the RGB slope to the method adopted for its computation. However, the value we find implies a metal content closer to the spectroscopic estimate $(-0.39 \pm 0.11$, Friel \& Janes 1993) than all the other photometric estimates (Janes 1979; Geisler 1987).

\section{Reddening}

In order to derive the interstellar extinction for NGC 2141, we have combined optical and IR photometry (500 stars in total), and constructed two color diagrams, namely $(J-$ $K)$ vs. $(V-K)$ and $(B-V)$ vs. $(V-K)$, which are shown in Figs. 5 and 6, respectively. Only MS stars are considered to avoid evolutionary effects. We superimposed a Zero Age MS (ZAMS) for the theoretical metal content $Z=0.007$, obtained by translating the observed $[\mathrm{Fe} / \mathrm{H}]$ by means of the relation:

$[\mathrm{Fe} / \mathrm{H}]=\log \frac{Z}{0.019}$

taken from Girardi et al. (2000b). In Fig. 5 the fit has been obtained by shifting the ZAMS with $E(J-K)=0.07$ and $E(V-K)=0.35$, which corresponds to a ratio $\frac{E(V-K)}{E(J-K)}=$ 5.0 , relatively close to the value 5.3 suggested by Cardelli et al. (1989). 


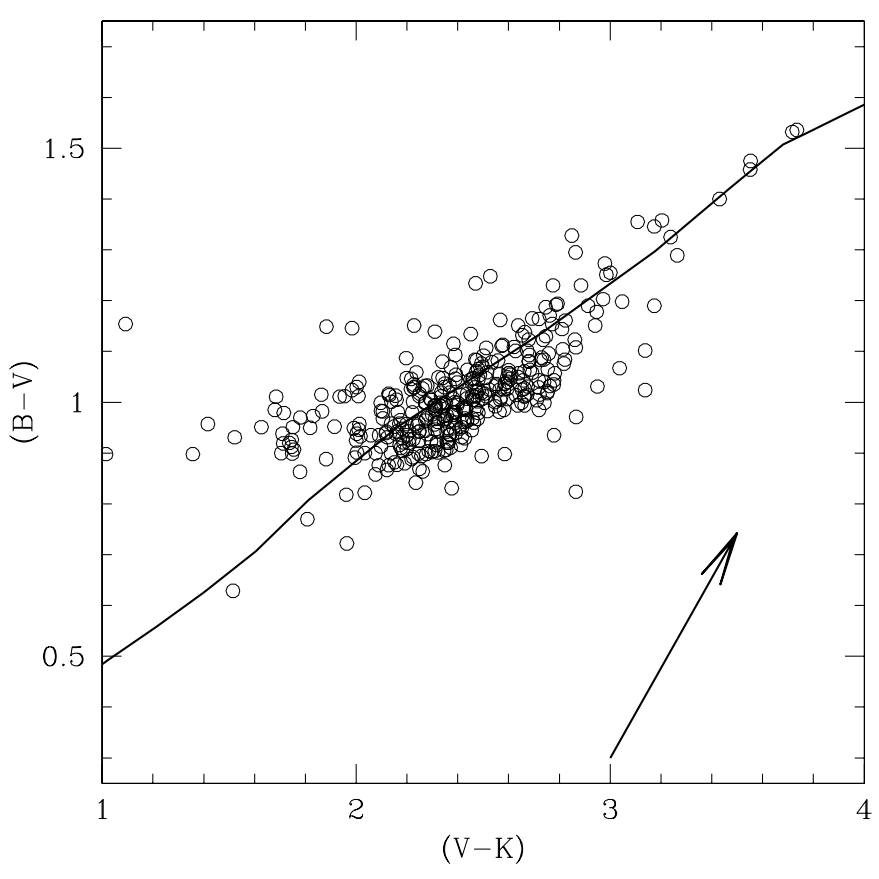

Fig. 6. Two color diagram for MS stars in NGC 2141. The solid line is a Zero Age MS for $[\mathrm{Fe} / \mathrm{H}]=-0.43$. In the lower right corner the reddening vector is shown. See text for details.

The fit in Fig. 6, on the other hand, has been achieved by shifting the ZAMS by $E(B-V)=0.40$ and $E(V-$ $K)=0.35$, whose ratio turns out to be $\frac{E(V-K)}{E(B-V)}=0.86$, in agreement with the value 0.87 from Cardelli et al. (1989). Although reasonable, these estimates are affected by the limitation that the reddening vector is almost parallel to the ZAMS, especially in the $(J-K)$ vs. $(V-K)$ plane. This is a minor problem in the plane $(B-V)$ vs. $(V-K)$, where we obtain a reddening value $E(B-V)=0.40$, not much different from $E(B-V)=0.35$ derived by Rosvick (1995). We must stress however that Rosvick (1995) did not take into account the metallicity of NGC 2141, which is lower than that of the Sun.

\section{Age and distance}

The knowledge of metallicity and reddening allows us to infer the distance and age of NGC 2141 by means of fitting with isochrones (Girardi et al. 2000b). The fit is shown in the two panels of Fig. 7, for the planes $V$ vs. $(B-V)$, and $K$ vs. $(J-K)$.

We have adopted the theoretical metallicity $Z=0.007$ derived above, and performed the fitting with a 2.5 Gyrs isochrone, which better matches the observational data. The criterion that guided us in performing this comparison was the simultaneous fit of the TO and the clump magnitudes. Since no membership exists for this cluster, it is not possible to exactly define the MS TO, which is populated also by unresolved binary stars, and interlopers. By using the reddening estimate derived in the previous Section, the apparent distance moduli $(m-M)_{K,(J-K)}$ and $(m-M)_{V,(B-V)}$ in the plots turn out to be 13.10 and

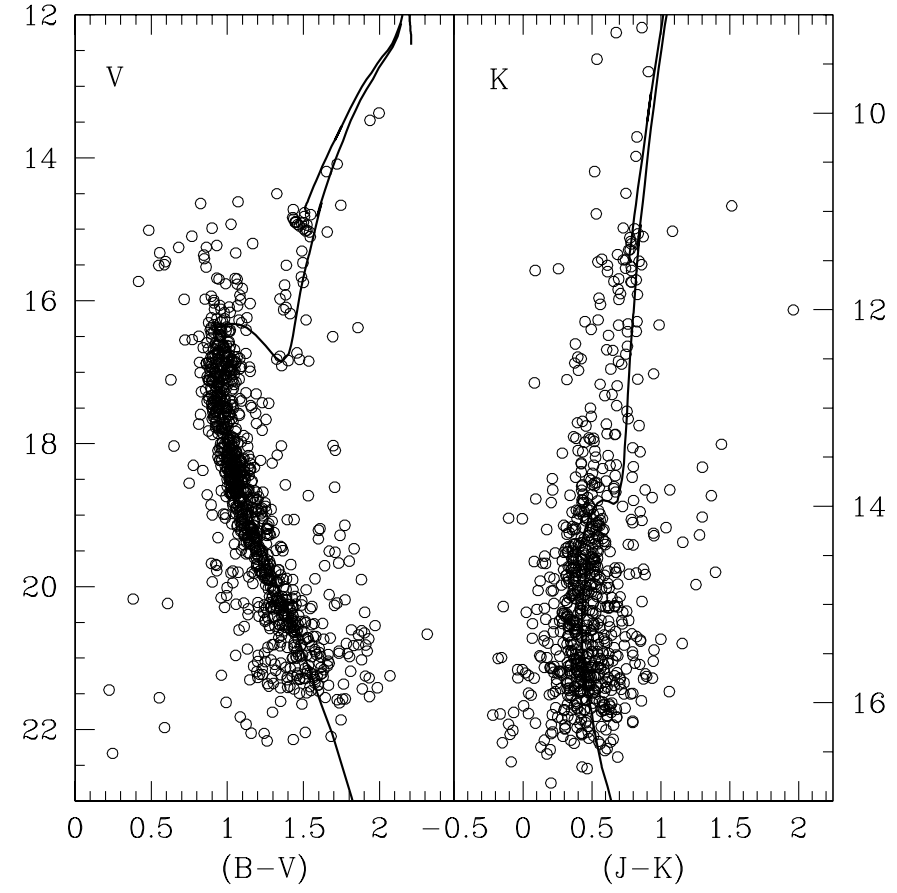

Fig. 7. Age determination for NGC 2141. Overimposed on the CMDs are $Z=0.007$ isochrones for an age of 2.5 Gyrs. The right panel shows the fit in the plane $K-(J-K)$, whereas the left panel shows the fit in the plane $V-(B-V)$. See text for details.

14.15, respectively. The latter value can also be obtained assuming that the mean clump magnitude is $M_{V}=0.80$ (Girardi et al. 1998). Once corrected, these values converge to the absolute distance modulus $(m-M)_{0}=12.90 \pm 0.15$. This value is in agreement within the errors with the Rosvick (1995) estimate.

NGC 2141 turns out to be $3.8 \pm 0.5 \mathrm{kpc}$ distant from the Sun, and about $12.0 \mathrm{kpc}$ away from the Galactic Center.

\section{Luminosity function}

In this section, we compute the differential Luminosity Function LF in the $V$ band. The data from the cluster have been previously corrected for completeness (see Carraro \& Ortolani 1994 for details). It resulted in $100 \%$ up to $V=$ 19 , then $85 \%$ at $19.7,73 \%$ at 20.2 and $66 \%$ at $V=20.7$. Fainter bins have not been considered because of the large amount of correction.

This LF is compared in Fig. 8 with theoretical ones for the same age range $(1.5,2.5$ and $3.5 \mathrm{Gyr})$ and metallicity $(z=0.007)$ as NGC 2141. The theoretical LFs have been calculated by assuming the IMF proposed by Kroupa et al. (1993), which is somewhat steeper than the classical Salpeter (1955) IMF, and best suited for the Solar Neighbourhood. It reads:

$\Phi(M)= \begin{cases}C_{k 1} M^{-0.5} & M<0.5 \\ C_{k} M^{-1.2} & 0.5<M<1 \\ C_{k} M^{-1.7} & M>1\end{cases}$ 


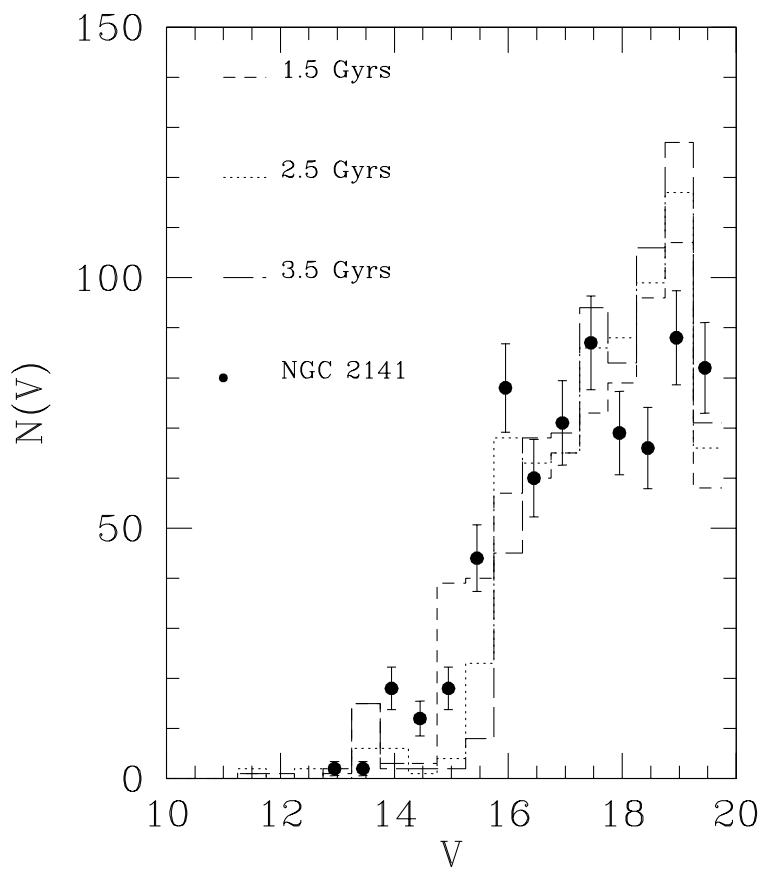

Fig. 8. Differential LF of MS stars in NGC 2141 (filled circles). Overimposed are three theoretical LFs for $Z=0.007$ and ages of 1.5, 2.5 and 3.5 Gyrs obtained by assuming the Kroupa et al. (1993) IMF. See the text for any detail.

where $C_{k 1}=0.48$ and $C_{k}=0.295$.

The comparison clearly shows that the agreement with theoretical models is good up to $M_{V}=5.0$. At fainter luminosities the theoretical models predict a higher number of stars, increasing with decreasing magnitudes. This occurs in the main sequence at about $1.0 M_{\odot}$, while the TO corresponds to about $1.4 M_{\odot}$.

It seems more reasonable to interpret these data as a more common mass segregation effect with low mass stars evaporated from the cluster center.

\section{Conclusions}

In this paper we have presented a detailed study of the poorly known intermediate age open cluster NGC 2141. By combining optical and IR photometry we have proved that NGC 2141 is a moderate age open cluster about $3 \mathrm{Gyr}$ old, intermediate in age between the NGC 752 and M 67.

By studying the color magnitude and two color diagrams, we have obtained estimates for the cluster metallicity, reddening and distance. In detail, we found that the color excesses $E(J-K)$ and $E(B-V)$ are 0.07, and 0.40, respectively, and that their ratios are in agreement with the standard values (Cardelli et al. 1989). The derived corrected distance modulus $(m-M)_{0}=12.90$ implies a distance of $3.8 \mathrm{kpc}$ from the Sun.

These findings are supported by the comparisons of the cluster LF with theoretical ones, from which we find also evidence of mass segregation below $M=1.0 M_{\odot}$.

Acknowledgements. This study has been financed by the Italian Ministry of University, Scientific Research and Technology (MURST) and the Italian Space Agency (ASI).

\section{References}

Bertelli, G., Bressan, A., \& Chiosi, C. 1992, ApJ, 392, 522

Burkhead, M. S., Burgess, R. D., \& Haisch, B. M. 1972, AJ, 77,661

Cardelli, J. A., Clayton, J. C., \& Mathis, J. S. 1989, ApJ, 345, 245

Carraro, G., \& Ortolani, S. 1994, A\&AS, 106, 573

Carraro, G., Vallenari, A., Girardi, L., \& Richichi, A. 1999a, A\&A, 343, 825

Carraro, G., Girardi, L., \& Chiosi, C. 1999b, MNRAS, 309, 430

Carraro, G., \& Vallenari, A. 2000, A\&AS, 142, 59

Friel, E. D. 1989, PASP, 101, 244

Friel, E. D., Liu, T., \& Janes, K. A. 1989, PASP, 101, 1105

Friel, E. D., \& Janes, K. A. 1993, A\&A, 267, 75

Geisler, D. 1987, AJ, 94, 84

Girardi, L., Groenewegen, M. A. T., Weiss, A., \& Salaris, M. 1998, MNRAS, 301, 149

Giraldi, L., Mermilliod, J.-C, \& Carraro, G. 2000a, A\&A, 354, 892

Girardi, L., Bressan, A., Bertelli, G., \& Chiosi, C. 2000b, A\&AS, 141, 371

Hunt, L. K., Mannucci, F., Testi, L., et al. 1998, AJ, 115, 2594

Janes, K. A. 1979, ApJS, 39, 135

Kassis, M., Janes, K. A., Friel, E. D., \& Phelps, R. L. 1997, AJ, 113, 1723

Kroupa, P., Tout, C. A., \& Gilmore, G. 1993, MNRAS, 262, 545

Landolt, A. U. 1992, AJ, 104, 340

Minniti, D. 1995, A\&AS, 113, 299

Rosvick, J. M. 1995, MNRAS, 277, 1379

Salpeter, E. E. 1955, ApJ, 129, 608

Tiede, G. P., Martini, P., \& Frogel, J. A. 1997, AJ, 114, 694

Vallenari, A., Richichi, A., Carraro, G., \& Girardi, L. 1999, A\&A, 349, 825

Vallenari, A., Carraro, G., \& Richichi, A. 2000, A\&A, 353, 147 